\title{
Secondary Hypogammaglobulinemia After Rituximab for Neuromyelitis Optica: A Case Report
}

\author{
Lara Farhat $^{1} \cdot$ Jasmeen Dara $^{2} \cdot$ Susan Duberstein ${ }^{3} \cdot$ Aliva De $^{1}$
}

Published online: 11 May 2018

(c) The Author(s) 2018

\begin{abstract}
A 17-year-old male with history of neuromyelitis optica and seizures presented to the pulmonology clinic for evaluation of recurrent pneumonias. $\mathrm{He}$ had received rituximab for the past 6 years. Over the past 2 years, he experienced four episodes of pneumonia. In between these episodes, he would improve briefly but continued to have daily cough that was productive with yellow phlegm. He also had recurrent rhinitis and sinusitis despite multiple antibiotic courses. Review of chest X-rays revealed localized right middle lobe and right lower lobe infiltrates. An extensive workup was performed, including computed tomography (CT) of the chest and bronchoscopy to rule out congenital lesions of the right lung and foreign body aspiration. Chest CT showed right lower lobe bronchiectasis. Flexible bronchoscopy with bronchoalveolar lavage showed normal anatomy with thick mucus secretions in the right lower lobe. Immunologic evaluation was performed and revealed low levels of immunoglobulin (Ig)-G, IgM, and IgA, which had declined since initiation of rituximab. Lymphocyte subset testing was remarkable
\end{abstract}

Lara Farhat

lafarhat@montefiore.org

1 Division of Pediatric Respiratory and Sleep Medicine, The Children's Hospital at Montefiore, Albert Einstein College of Medicine, 3415 Bainbridge Ave, Bronx, New York 10467, USA

2 Division of Allergy and Immunology, Department of Pediatrics, The Children's Hospital at Montefiore, Albert Einstein College of Medicine, 3415 Bainbridge Ave, Bronx, New York 10467, USA

3 Division of Neurology, Department of Pediatrics, The Children's Hospital at Montefiore, Albert Einstein College of Medicine, 3415 Bainbridge Ave, Bronx, New York 10467, USA for low cluster of differentiation (CD)-19. He was referred to allergy and immunology and was initiated on immunoglobulin-replacement therapy (IGRT) for acquired hypogammaglobulinemia secondary to rituximab. There was marked clinical improvement after initiation of IGRT.

\section{Key Points}

Rituximab may induce hypogammaglobulinemia when used for autoimmune diseases.

Consideration of secondary immunodeficiency is important when evaluating patients for recurrent pneumonias who are also receiving cluster of differentiation (CD)-19 B-cell depleting immunotherapy.

\section{Introduction}

The etiology of recurrent pneumonias in children may be multifactorial and can include, but is not limited to, congenital malformations of the lung, foreign body aspiration, atypical infections, underlying immune disorder, and aspiration. We present a case of recurrent pneumonias in a patient receiving rituximab for neuromyelitis optica. 


\section{Case Report}

A 17-year-old male with history of neuromyelitis optica (NMO) and seizures presented to the pediatric pulmonology clinic for evaluation of recurrent pneumonias. He had been diagnosed with NMO with positive antibodies at 9 years of age after acute vision loss in the left eye. At that time, he was started on azathioprine and chronic steroids. At 2 years after initial presentation, he was initiated on rituximab after his condition worsened and he developed blurry vision in the contralateral eye. All other immunosuppressive medications were discontinued following initiation of rituximab. He was receiving rituximab $1000 \mathrm{mg}$ every 6 months for 6 years before evaluation by pediatric pulmonology. At his pulmonary evaluation, he presented with a history of four pneumonias over the past 2 years, two of which required admission for intravenous antibiotics and two of which were treated as an outpatient with oral antibiotics. There was no previous history of tonsillitis, ear infections, sinusitis, or recurrent pneumonias.

In between his recent episodes of pneumonia, he would improve briefly but continued to have daily productive cough with yellow phlegm. He also had recurrent rhinitis and sinusitis despite multiple antibiotic courses. Additionally, he had a history of positive respiratory $M y$ cobacterium avium-intracellulare (MAI) polymerase chain reaction (PCR) test at the time of his initial pneumonia that was not treated because it was a single positive test. His physical exam was notable for normal vital signs, erythema and opacity of the right tympanic membrane, nasal congestion, and decreased breath sounds on the right side with crackles. Review of previous chest radiographs revealed localized right middle lobe and right lower lobe infiltrates. An extensive workup was subsequently initiated.

A chest computed tomography (CT) scan revealed localized bronchiectasis and mucoid impaction, as shown in Fig. 1. Flexible bronchoscopy with bronchoalveolar lavage (BAL) showed normal anatomy with thick mucus secretions in the right lower lobe. BAL fluid cell count was notable for $100 \%$ neutrophils. BAL bacterial culture was positive for Streptococcus viridans but negative for acid fast bacilli. BAL cytology was negative for lipid-laden and hemosiderin-laden macrophages.

Quantitative immunoglobulin panel revealed low levels of $\operatorname{IgA}, \mathrm{IgG}$, and IgM. Prior labs revealed a gradual decline of immunoglobulins over the past few years (Table 1). This decline was attributed to secondary hypogammaglobulinemia following rituximab administration. Baseline immunoglobulin levels prior to initiation of rituximab were normal, which indicated against a primary immunodeficiency. Lymphocyte subset testing was remarkable for high cluster of differentiation (CD)-8 count (1622 cells $/ \mu \mathrm{l}$ [54\%]) and low CD19 level (1 cell/ $\mu \mathrm{l}[1 \%])$.

Because of the CT findings and clinical symptoms, airway clearance was initiated. He was then referred to allergy and immunology and was started on intravenous immunoglobulin replacement therapy (IGRT) for acquired hypogammaglobulinemia secondary to rituximab. Marked clinical improvement of his upper and lower respiratory disease was noted after initiation of IGRT.

\section{Discussion}

NMO, also known as Devic disease, is an autoimmune demyelinating disease of the central nervous system that selectively affects the spinal cord and optic nerve. B-cellmediated humoral immunity has been implicated in the pathogenesis of the disease [1], leading to resultant primary injury of astrocytes arbitrated by the formation of aquaporin-4 antibodies [2].

Rituximab is a chimeric monoclonal antibody that targets the CD20 antigen on B cells to decrease the frequency and severity of attacks in patients with NMO. Treatment results in precursor and mature B-cell depletion as a result of complement-dependent cytotoxicity or antibody-dependent cellular cytotoxicity, after which the effects can
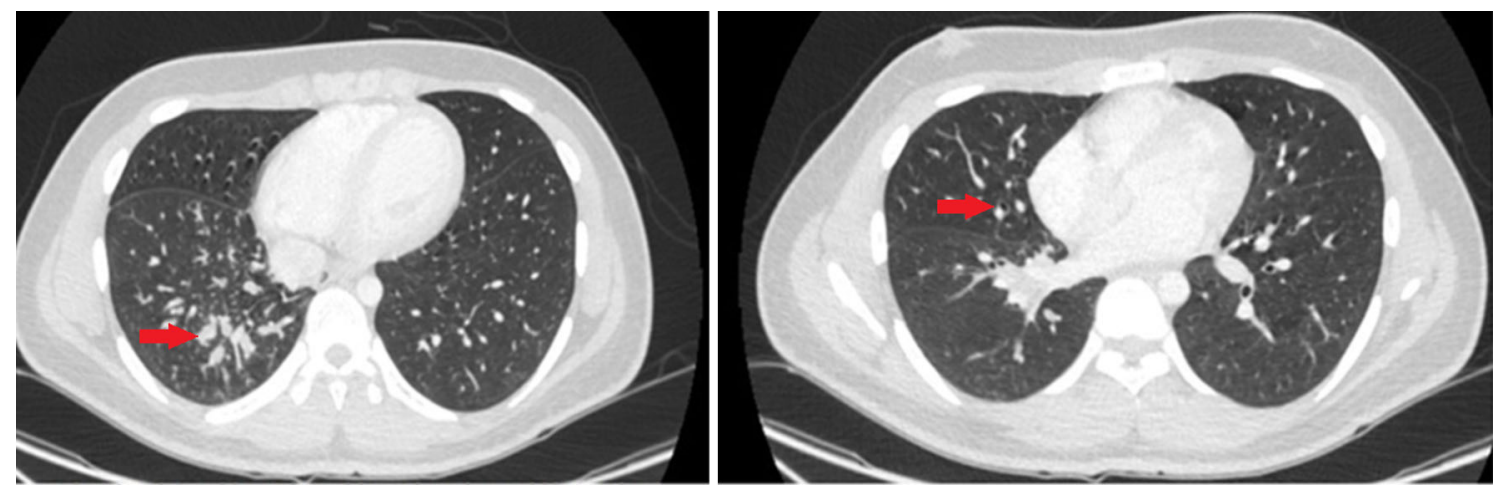

Fig. 1 Computed tomography (CT) chest with arrows showing mid-zone mucoid impaction and bronchiectasis 
Table 1 Immunoglobulin levels at presentation, 3 and 6 years before pulmonary visit

\begin{tabular}{lcccc}
\hline & Reference ranges $(\mathrm{mg} / \mathrm{dl})$ & Current levels & 3 years prior & 6 years prior \\
\hline IgG & $844-1912$ & 564 & 620 & 835 \\
IgA & $68-423$ & 32.2 & 51 & 213 \\
IgM & $50-196$ & $<4.3$ & 8 & 63 \\
\hline
\end{tabular}

Ig immunoglobulin persist for 6-9 months [3]. The function of rituximab is through two stages. In the first stage, all CD19+ B cells, including regulatory and pro-inflammatory, are deleted [1]. $\mathrm{T}-\mathrm{B}$ cell interaction is interrupted, causing a decline in the production of new autoantibody-producing plasma cell precursors [1]. In the second stage, B-lymphocyte reconstitution occurs [1]. After treatment, there is a predominance of protective regulatory $B$ lymphocytes over pathogenic memory B lymphocytes, which are predominant prior to treatment. The most commonly reported adverse effects of rituximab therapy include infusion-related effects [4], urogenital infection, and varicella zoster virus reactivation [5], although some deaths have been reported secondary to extensive myelitis [6] and urosepsis [5]. Another rare but serious complication is rituximabassociated lung injury (RALI). Presenting symptoms have been reported to include fever, chronic cough, hypoxemia, weakness, and dyspnea [7]. CT of the chest typically shows bilateral ground glass opacities [7]. It is a diagnosis of exclusion following a negative infectious workup [7].

Hypogammaglobulinemia after rituximab has been most commonly reported in the setting of pre-existing immune deficiency, autologous stem cell transplant, and B-cell lymphoma [8]. In these patients, the number of doses of rituximab administered was correlated with developing symptomatic hypogammaglobulinemia [8]. Rituximab has also been reported to induce neutropenia and thrombocytopenia [9]. Less commonly, hypogammaglobulinemia has occurred secondary to rituximab used for autoimmune diseases such as idiopathic thrombocytopenic purpura and rheumatoid arthritis [10, 11]. As such, baseline immunoglobulin levels should be checked, and regular monitoring of immunoglobulin levels has been recommended, with consideration of intravenous immunoglobulin replacement for symptomatic infections [12]. In our patient, immunoglobulin levels began gradually declining approximately 1 year after initiation of rituximab, and he became clinically symptomatic about 3 years after initiation of this therapy. A Naranjo Adverse Drug Reaction Probability score of 9 was obtained for our case, which indicates a definite relationship between rituximab administration and subsequent immunodeficiency [13].

In children who present with recurrent pneumonias, a multifactorial etiology must be considered and can include, but is not limited to, congenital malformations of the lung, foreign body aspiration, atypical infections, underlying immune disorder, and aspiration. Imaging can help exclude some of these etiologies, such as congenital lung malformations. Bronchoscopy is helpful for general surveillance of airway anatomy and ruling out foreign body aspiration. BAL samples can be sent for cultures as well as cytology. The presence of hemosiderin-laden and lipid-laden macrophages can be markers for alveolar hemorrhage and aspiration, respectively. A thorough immunologic workup is also warranted for these patients, including cell count, immunoglobulin levels, and lymphocyte subsets.

The presence of recurrent pneumonias and chronic cough in any patient should lead to a suspicion of bronchiectasis. In predisposed individuals, chronic inflammation may lead to airway obstruction and damage. This is best explained by a cycle proposed by Cole [14], where bacterial colonization leads to neutrophil inflammation, which in turn leads to airway destruction and distortion, or bronchiectasis. These airway structural abnormalities lead to mucus stasis, which continues to harbor bacterial colonization and perpetuates the vicious cycle [14]. High-resolution CT is the diagnostic test of choice for bronchiectasis [15]. Initiation of an airway clearance regimen is important in slowing down this cycle.

\section{Conclusion}

Although not frequently reported, hypogammaglobulinemia can develop as a consequence of rituximab therapy for autoimmune diseases, such as NMO. Regular monitoring of immunoglobulin levels in patients receiving rituximab is therefore warranted, with consideration of immunoglobulin replacement for symptomatic infections. Moreover, consideration of secondary immunodeficiency is important when evaluating patients for recurrent pneumonias who are also receiving CD19 B-cell depleting immunotherapy.

\section{Compliance with Ethical Standards}

Funding No financial support was received for the conduct of this study or preparation of this manuscript.

Consent for Publication Written informed consent was obtained from the patient for the publication of this case report and the 
accompanying images. A copy of the written consent may be requested for review from the corresponding author.

Conflict of interest Lara Farhat, Jasmeen Dara, Susan Duberstein, and Aliva De have no conflicts of interest.

Open Access This article is distributed under the terms of the Creative Commons Attribution-NonCommercial 4.0 International License (http://creativecommons.org/licenses/by-nc/4.0/), which permits any noncommercial use, distribution, and reproduction in any medium, provided you give appropriate credit to the original author(s) and the source, provide a link to the Creative Commons license, and indicate if changes were made.

\section{References}

1. Etemadifar M, Salari M, Mirosayyeb O, Serati M, Nikkah R, Askari M, Fayyazi E. Efficacy and safety of rituximab in neuromyelitis optica: review of evidence. $J$ Res Med Sci. 2017;22(18):2.

2. Hoftberger R, Lassmann H. Inflammatory demyelinating diseases of the central nervous system. Handb Clin Neurol. 2017; 145:263-83.

3. Evangelopoulos ME, Andreadou E, Koutsis G, Koutoulidis V, Anagnostouli M, Katsika P, Evangelopuols DS, Evdokimidis I, Kilidireas C. Treatment of neuromyelitis optica and neuromyelitis optica spectrum disorders with rituximab using a maintenance treatment regimen and close CD19 B cell monitoring. A six-year follow-up. J Neurol Sci. 2017;372:92-6.

4. Fernández-Megía MJ, Casanova-Estruch B, Pérez-Miralles F, Ruiz-Ramos J, Alcalá-Vicente C, Poveda-Andrés JL. Clinical evaluation of rituximab treatment for neuromyelitis optica. Neurologia. 2015;30:461-4.

5. Pellkofer HL, Krumbholz M, Berthele A, Hemmer B, Gerdes LA, Havla J, Bittner R, Canis M, Meinl E, Hohlfeld R, Kuempfel T. Long-term follow up of patients with neuromyelitis optica after repeated therapy with rituximab. Neurology. 2011;76(15):1310-5.
6. Zéphir H, Bernard-Valnet R, Lebrun C, Outteryck O, Audoin B, Bourre B, Pittion S, Wiertlewski S, Ouallet JC, Neau JP, Ciron J, Clavelou P, Marignier R. Brassat. Rituximab as first-line therapy in neuromyelitis optica: efficiency and tolerability. J Neurol. 2015;262(10):2329-35.

7. Bitzan M, Anselmo M, Carpineta L. Rituximab (B-cell depleting antibody) associated lung injury (RALI): a pediatric case and systematic review of the literature. Pediatr Pulmonol. 2009;44(9):922-34.

8. Casulo C, Maragulia J, Zelenetz AD. Incidence of hypogammaglobulinemia in patients receiving rituximab and the use of intravenous immunoglobulin for recurrent infections. Clin Lymphoma Myeloma Leuk. 2013;13:106-11.

9. Cattaneo C, Spedini P, Casari S, Re A, Tucci A, Borlenghi E, Ungari M, Ruggeri G, Rossi G. Delayed-onset peripheral blood cytopenia after rituximab: frequency and risk factor assessment in a consecutive series of 77 treatments. Leuk Lymphoma. 2006;47:1013-7.

10. Cooper N, Davies EG, Thrasher AJ. Repeated courses of rituximab for autoimmune cytopenias may precipitate profound hypogammaglobulinaemia requiring replacement intravenous immunoglobulin. Br J Haematol. 2009;146(1):120-2.

11. Keystone E, Fleischmann R, Emery P, Furst DE, van Vollenhoven R, Bathon J, Dougados M, Baldassare A, Ferraccioli G, Chubick A, Udell J, Cravets MW, Agarwal S, Cooper S, Magrini F. Safety and efficacy of additional courses of rituximab in patients with active rheumatoid arthritis: an open-label extension analysis. Arthritis Rheum. 2007;56(12):3896-908.

12. Cooper N, Arnold DM. The effect of rituximab on humoral and cell mediated immunity and infection in the treatment of autoimmune diseases. Br J Haematol. 2010;149(1):3-13.

13. Naranjo CA, Busto U, Sellers EM, Sandor P, Ruiz I, Roberts EA, Janecek E, Domecq C, Greenblatt DJ. A method for estimating the probability of adverse drug reactions. Clin Pharmacol Ther. 1981;30(2):239-45.

14. Cole PJ. Inflammation: a two-edged sword-the model of bronchiectasis. Eur J Respir Dis Suppl. 1986;147:6-15.

15. Pasteur MC, Bilton D, Hill AT. British thoracic society bronchiectasis non-CF guideline group. British Thoracic Society guideline for non-CF bronchiectasis. Thorax. 2010;65:i1-58. 